\title{
Article \\ Wavelet Spectra of a Chirped Gaussian Pulsed-Driven Harmonic Oscillator
}

\author{
R. A. Alharbey ${ }^{*}+\boldsymbol{t}^{-}$and H. Abusamra ${ }^{\dagger}$
}

Mathematics Department, Faculty of Science, King Abdulaziz University, P.O. Box 80200, Jeddah 21589, Saudi Arabia; lolobash26@gmail.com

* Correspondence: Rallehabi@kau.edu.sa

† These authors contributed equally to this work.

\begin{abstract}
We investigate the nature of spectral lines of emitted radiation due to of a non-dissipative single-mode quantized harmonic oscillator ( $\mathrm{HO}$ ) with train of n-chirped Gaussian pulses. Specifically, we analyze the transient emitted spectrum through two window wavelet functions of the radiation detector, namely the Haar wavelet and Morlet wavelet. Computational display of the exact analytical results shows how the driving pulse parameters (strength and number of pulses, chirping, repetition time) act as control knobs to shape the detected emitted spectrum as desired.
\end{abstract}

Keywords: harmonic oscillator; chirped gaussian laser pulses; haar and morlet wavelet spectra

check for updates

Citation: Alharbey, R.A.; Abusamra, H. Wavelet Spectra of a Chirped Gaussian Pulsed-Driven Harmonic Oscillator. Mathematics 2021, 9, 939. https://doi.org/10.3390/math9090939

Academic Editors: Rami Ahmad El-Nabulsi and Luigi Rodino

Received: 22 March 2021

Accepted: 21 April 2021

Published: 23 April 2021

Publisher's Note: MDPI stays neutral with regard to jurisdictional claims in published maps and institutional affiliations.

Copyright: (c) 2021 by the authors. Licensee MDPI, Basel, Switzerland. This article is an open access article distributed under the terms and conditions of the Creative Commons Attribution (CC BY) license (https:// creativecommons.org/licenses/by/ $4.0 /)$.

\section{Introduction}

Driven quantized harmonic oscillator $(\mathrm{HO})$ is a fundamental model of radiation-radiation interaction in the field of nonlinear and quantum optics, e.g., $[1,2]$. The case when the driving field is a pulsed laser has been investigated regarding the fluorescence Fourier transform (FT) [3-7] and wavelet transform (WT) [7,8] spectra of the emitted radiation for various shapes of the laser pulse. Briefly speaking, FT analysis is a time-averaging process over the entire time interval of the detected signal, and in turn some localized-time structure may be lost due to this time-averaging [9]. On the other hand, time-localized structure of different frequency components in the detected signal can be dealt with through adjustment of the time width of the detecting "mother window function". This is known as WT analysis, in which wavelets of high frequencies are narrower and vice versa [9]. Transient wavelet spectral analysis of the emitted radiation of a single 2-level atom driven by a rectangular pulse has been given in the cases of Morlet and Mexican hat [10] and Haar [11] wavelet window functions. Also, for the case of 2-level atom, Haar wavelet spectrum has been investigated where the driving pulse is of exponentially decaying shape [12] and of $\sin ^{2}$-shape [13].

In this paper, we expand our results in [5] for the model of a single non-dissipative $\mathrm{HO}$ driven by a field that has the shape of a train of n-Chirped Gaussian pulses and calculate the wavelet transform spectra with two different wavelet window functions, namely Haar wavelet (HW) and Morlet wavelet (MW) windows. We investigate the WT spectra for cases of initial vacuum and number states of the HO.

The paper is presented as follows: in Section 2, we present the model equations for the driven $\mathrm{HO}$ with the expression for the average auto-correlation function of the $\mathrm{HO}$ amplitude operators. Analytical expressions for the transient WT spectra are derived in Section 3 for the two cases of HW and MW window functions. In Section 4, we present the computational results for both WT spectra, with initial vacuum and number states of the $\mathrm{HO}$, followed by summary in Section 5 . 


\section{Model Equations and Correlation functions}

The Hamiltonian model of a single-mode HO of frequency $\omega_{0}$ driven by a laser pulse of complex envelope $f(t)$ and of oscillating frequency $\omega_{L}$, within the rotating wave approximation (RWA) and in the absence of damping processes (in units of $\hbar=1$ ) has the form $[14,15]$,

$$
H=\omega_{0} \hat{a}^{\dagger} \hat{a}+\Omega_{0}\left(f(t) \hat{a}^{\dagger} e^{-i \omega_{L} t}+\text { h.a. }\right) .
$$

The notations are: $\Omega_{0}$ is the Rabi frequency associated with the driving pulse (assumed real), the annihilation and creation HO operators, $\hat{a}$ and $\hat{a}^{\dagger}$, respectively, obey the boson commutation relation $\left[\hat{a}, \hat{a}^{\dagger}\right]=1$, and $h \cdot a$ stands for Hermitian adjoint.

In the case, n-chirped Gaussian pulse, the envelope $f(t)$ has the form [5],

$$
f(\tau)=\sum_{m=1}^{n} e^{-(1+i C)\left(\tau-(m-1) \tau_{R}\right)^{2}}
$$

where $\tau=\frac{t}{\tau_{0}}$ is the normalized time, $\tau_{0}$ is the full width of one pulse at $\frac{1}{2}$-maximum, $\tau_{R}=\frac{T_{R}}{\tau_{o}}$ is the normalized repetition time and $C$ is the chirp parameter.

Heisenberg equations of motion for the $\mathrm{HO}$ operators according to the Hamiltonian (1) are of the form,

$$
\begin{aligned}
\dot{\hat{a}} & =-i \omega_{0} \hat{a}-i \Omega_{0} f(t) e^{-i \omega_{L} t} \\
\dot{a}^{\dagger} & =i \omega_{0} \hat{a}^{\dagger}+i \Omega_{0} f^{*}(t) e^{i \omega_{L} t}
\end{aligned}
$$

The solutions of (2) for arbitrary initial time $t_{o}$ are of the form,

$$
\begin{aligned}
\hat{a}(t) & =e^{-i \omega_{0} t}\left(e^{i \omega_{0} t_{0}} \hat{a}\left(t_{o}\right)-i \Omega_{0} I(t)\right) \\
& =e^{-i \omega_{0} t} \hat{A}(t) \\
\hat{a}^{\dagger}(t) & =e^{i \omega_{0} t} \hat{A}^{\dagger}(t),
\end{aligned}
$$

where

$$
\begin{aligned}
& \hat{A}(t)=e^{i \omega_{0} t_{0}} \hat{a}\left(t_{0}\right)-i \Omega_{0} I(t) \\
& I(t)=\int_{t_{0} \rightarrow-\infty}^{t} f\left(t^{\prime}\right) e^{-i \Delta t^{\prime}} d t^{\prime}
\end{aligned}
$$

and have taken $t_{0} \rightarrow-\infty$, since Gaussian pulses are of smooth switch-on shape, with $\Delta=\omega_{L}-\omega_{0}$ is the atomic detuning. The quantity $I(t)$ in (5) in this case is given by [5],

$$
I(t)=\sqrt{\frac{\pi}{a}} \frac{\tau_{o}}{2} \sum_{m=1}^{n} e^{-(1+i C)(m-1)^{2} \tau_{R}^{2}} e^{\frac{b_{m}^{2}}{a}}\left[1+\operatorname{erf}\left(c_{1} t+b_{m} / \sqrt{a}\right)\right],
$$

where

$$
\begin{aligned}
a & =1+i C \\
b_{m} & =\frac{a}{2}\left(\frac{\left(i \Delta_{o}\right)}{a}-2(m-1) \tau_{R}\right) \\
c_{1} & =\frac{\sqrt{a}}{\tau_{o}}, \Delta_{o}=\Delta \tau_{o},
\end{aligned}
$$


and $\operatorname{erf}(\mathrm{z})=\frac{2}{\sqrt{\pi}} \int_{0}^{z} e^{-z^{\prime 2}} d z^{\prime}$ is the error function of complex argument [16]. The average value of the auto-correlation function $\left\langle\hat{a}^{\dagger}\left(t_{1}\right) \hat{a}\left(t_{2}\right)\right\rangle$ can be calculated from the solutions (4),

$$
\begin{aligned}
\left\langle\hat{a}^{\dagger}\left(t_{1}\right) \hat{a}\left(t_{2}\right)\right\rangle & =e^{i \omega_{o}\left(t_{1}-t_{2}\right)}\left(\left\langle\hat{a}^{\dagger}\left(t_{o}\right) \hat{a}\left(t_{o}\right)\right\rangle+\Omega_{o}^{2} I^{*}\left(t_{1}\right) I\left(t_{2}\right)-i \Omega_{o} \bar{a}_{o}^{*} I\left(t_{2}\right),\right. \\
& \left.+i \Omega_{o} \bar{a}_{o} I^{*}\left(t_{1}\right)\right),
\end{aligned}
$$

where $\bar{a}_{o}=e^{-i \omega_{0} t_{0}}\left\langle\hat{a}\left(t_{0}\right)\right\rangle$ is the initial average value of the HO (amplitude) operator, $\hat{a}\left(t_{0}\right)$ modified by the phase factor $e^{-i \omega_{0} t_{0}}$. In the case of initial number state $\left|n_{0}\right\rangle$ of the $\mathrm{HO}$, where $\left\langle\hat{a}^{\dagger}\left(t_{o}\right) \hat{a}\left(t_{o}\right)\right\rangle=n_{0}, \bar{a}_{o}=0$, Equation (8) has the form,

$$
\left\langle\hat{a}^{\dagger}\left(t_{1}\right) \hat{a}\left(t_{2}\right)\right\rangle_{n o}=e^{i \omega_{o}\left(t_{1}-t_{2}\right)}\left(n_{o}+\Omega_{o}^{2} I^{*}\left(t_{1}\right) I\left(t_{2}\right)\right) .
$$

As seen from the dynamical solutions for the HO amplitudes (4), (5) and the autocorrelation functions, Equation (8), that the Rabi frequency parameter $\Omega_{0}$ does not contribute to any oscillatory behavior, i.e., no induced Rabi oscillations. This is unlike the case of driven atomic systems (of finite number of energy levels), e.g., [3,10-13], where Rabi oscillations (particularly in the strong field limit) manifest itself in the bouncing of atoms between its energy levels. Oscillatory behavior in our driven $\mathrm{HO}$ model are soloy due to pulse shape and wavelet window functions parameters.

\section{Transient Wavelet Spectra: Analytical Results}

The transient spectrum of the scattered radiation is given by [17].

$$
S\left(t, \omega_{f}, \Gamma\right)=2 \Gamma \int_{t_{0} \rightarrow-\infty}^{t} d t_{1} \int_{t_{0} \rightarrow-\infty}^{t} d t_{2} H\left(t-t_{1}\right) H^{*}\left(t-t_{2}\right)\left\langle\hat{a}^{\dagger}\left(t_{1}\right) \hat{a}\left(t_{2}\right)\right\rangle .
$$

For the response (i.e., window) function of the photo-detector (of width $\Gamma$ and frequency $\left.\omega_{f}\right)$ is given by [8],

$$
H\left(t-t_{1}\right)=\psi\left(t-t_{1}\right) e^{\left(i \omega_{f}-\Gamma\right)\left(t-t_{1}\right)}
$$

where $\psi\left(t-t_{1}\right)$ is the mother wavelet function. Here, we consider the following two cases of wavelet functions, $\psi\left(t-t_{1}\right)$.

\subsection{Haar Wavelet Spectrum}

The mother wavelet function in this case has the bi-polar step shape $[9,18]$

$$
\psi\left(t-t_{1}\right)=2^{\frac{j_{0}}{2}}\left\{\begin{array}{l}
-1, \alpha \leq t_{1}<\beta \\
1, \beta \leq t_{1}<\gamma \\
0, \text { otherwise }
\end{array}\right.
$$

with $\alpha, \beta, \gamma$ are scaled time-dependent variables given by,

$$
\begin{aligned}
& \alpha=t-2^{-j_{o}}\left(1+k_{o}\right) \\
& \beta=t-2^{-j_{o}}\left(\frac{1}{2}+k_{o}\right) \\
& \gamma=t-2^{-j_{o}} k_{o}
\end{aligned}
$$

and $j_{0}, k_{o}$ are the dilation and shift parameters, respectively.

The HW, although has a good time resolution, it has no frequency resolution, i.e., continuous signals are not approximated very well due to its time domain discontinuity $[18,19]$. 
Using Equation (12) into (10) and the use of Equation (9) into (8), we get the wavelet spectrum, in the case of initial number state $\left|n_{o}\right\rangle$ of the $\mathrm{HO}$ into the form,

$$
S_{H, n_{o}}(t, D, \Gamma)=2^{j_{o}+1} \Gamma e^{-2 \Gamma t}\left[n_{o}\left|J_{1, H}(t)\right|^{2}+\Omega_{o}^{\prime 2}\left|J_{2, H}(t)\right|^{2}\right]
$$

The expressions for the $J_{1, H}(t), J_{2, H}(t)$ are given by,

$$
\begin{gathered}
J_{1, H}(t)=\left(\int_{\alpha}^{\beta}-\int_{\beta}^{\gamma}\right) e^{(\Gamma-i D) t^{\prime}} d t^{\prime} \\
=\frac{e^{(\Gamma-i D) \tau_{k o}}}{(\Gamma-i D)} \alpha_{1}, \\
J_{2, H}(t)=\left(\int_{\alpha}^{\beta}-\int_{\beta}^{\gamma}\right) e^{(\Gamma-i D) t^{\prime}} I^{*}\left(t^{\prime}\right) d t^{\prime} \\
=\sqrt{\frac{\pi}{a^{*}}} \frac{1}{2} \sum_{m=1}^{n} e^{-(1-i c)(m-1)^{2} \tau_{R}^{2}} e^{\left(b_{m}^{2} / a\right)^{*}}\left(J_{1, H}(t)+T_{1, m}(t)\right),
\end{gathered}
$$

with,

$$
\begin{aligned}
T_{1, m}(t) & =\frac{e^{-c_{2}^{*} d}}{c_{1}^{*} d}\left[e^{\left(c_{1}^{*} \tau_{k_{o}}+c_{2}^{*}\right) d} \alpha_{2}-e^{\frac{d^{2}}{4}} \alpha_{3}\right] \\
\alpha_{1} & =2 e^{-2^{-j_{o}-1}(\Gamma-i D)}-e^{-2^{-j_{o}}(\Gamma-i D)}-1 \\
\alpha_{2} & =2 e^{-2^{-j_{o}-1}} \operatorname{erf}\left(c_{1}^{*}\left(\tau_{k_{o}}-2^{-j_{o}-1}\right)+c_{2}^{*}\right)-2^{-j_{o}} \operatorname{erf}\left(c_{1}^{*}\left(\tau_{k_{o}}-2^{-j_{o}}\right)+c_{2}^{*}\right)-\operatorname{erf}\left(c_{1}^{*} \tau_{k_{o}}+c_{2}^{*}\right) \\
\alpha_{3} & =2 \operatorname{erf}\left(c_{1}^{*}\left(\tau_{k_{o}}-2^{-j_{o}-1}\right)+c_{2}^{*}-\frac{d}{2}\right)-\operatorname{erf}\left(c_{1}^{*}\left(\tau_{k_{o}}-2^{-j_{o}}\right)+c_{2}^{*}-\frac{d}{2}\right)-\operatorname{erf}\left(c_{1}^{*} \tau_{k_{o}}+c_{2}^{*}-\frac{d}{2}\right) \\
c_{2} & =b_{m} / \sqrt{a}, d=\frac{\Gamma-i D}{c_{1}^{*}}, \tau_{k} o=\tau-2^{-j_{o}} k_{o}, D=\omega_{f}-\omega_{o}, \Omega_{o}^{\prime}=\Omega_{o} \tau_{o}
\end{aligned}
$$

\subsection{Morlet Wavelet Spectrum}

The mother wavelet function $\psi_{M}\left(t-t_{1}\right)$ is in this case $[9,18]$,

$$
\psi_{M}\left(t-t_{1}\right)=e^{-i \omega_{M}\left(\frac{t-k}{j}-t_{1}\right)} e^{-\left(\frac{t-k}{j}-t_{1}\right)^{2} / 2 \sigma^{2}},
$$

where $j, k$ are the dilation and shift parameters, respectively, and $\omega_{M}$ is the central frequency of the mother wavelet and the $\sigma$ is full width at $\frac{1}{2}$-maximum of the Gaussian envelope. In what follows, for the Morlet spectrum, we put $\sigma=1$ which means the parameters $t, \Omega_{0}, \omega_{0}, \omega_{f}, \omega_{M}, \Gamma$ are normalized to $\sigma$. The Morlet (or Gaussian) wavelet is much suitable with signal analysis for two aspects: its oscillatory and decay nature allow for good frequency and time resolutions, respectively $[18,19]$.

Using Equations (11), (9), (16) in Equation (10) we get the analytical expression for the Morlet wavelet spectrum with initial number state $\left|n_{o}\right\rangle$ of the $\mathrm{HO}$ into the form,

$$
S_{M, n_{o}}\left(t, D_{1}, \Gamma\right)=2 \Gamma e^{-2 \Gamma t}\left|e^{\lambda_{1}(t)}\right|\left[n_{o}\left|J_{1, M}(t)\right|^{2}+\Omega_{o}^{2}\left|J_{2, M}(t)\right|^{2}\right]
$$


The quantities for the $J_{1, M}, J_{2, M}$ are given by,

$$
\begin{aligned}
& J_{1, M}(t)=\sqrt{\frac{\pi}{2}}\left(1-\operatorname{erf}\left(\lambda_{2}(t)\right)\right) \\
& J_{2, M}(t)=\frac{1}{2} \sqrt{\frac{\pi}{a^{*}}} \sum_{m=1}^{n} e^{-(1-i C)(m-1)^{2} \tau_{R}^{2}} e^{\left(b_{m}^{2} / a\right)^{*}}\left(J_{1, M}(t)+T_{2, m}(t)\right), \\
& T_{2, m}(t)=\sqrt{2} \int_{-\lambda_{2}(t)}^{\infty} e^{-t^{\prime 2}} \operatorname{erf}\left(-\sqrt{2} c_{1}^{*} t^{\prime}+\lambda_{3}\left(t^{\prime}\right)\right) d t^{\prime}
\end{aligned}
$$

where

$$
\begin{aligned}
& \lambda_{1}(t)=2\left(\Gamma-i D_{M}\right)(t-k) / j+\left(\Gamma-i D_{M}\right)^{2} \\
& \lambda_{2}(t)=\frac{t(1-j)-k}{j}+\frac{1}{\sqrt{2}}\left(\Gamma-i D_{M}\right) \\
& \lambda_{3}(t)=c_{1}^{*}(t-k) / j+c_{1}^{*}\left(\Gamma-i D_{M}\right)+\left(b_{m} / \sqrt{a}\right)^{*}
\end{aligned}
$$

with the (normalized) detector's detuning $D_{M}=\omega_{f}-\left(\omega_{o}+\omega_{M}\right)$. The integral in (18c) is best treated numerically.

\section{Computational Results}

Here, we plot the analytical formulas for the wavelet spectra $S_{H}\left(D_{H}\right)=S_{H}\left(t, D_{1}, \Gamma\right)$ and $S_{M}\left(D_{M}\right)=S_{M}\left(t, D_{1}, \Gamma\right)$ normalized to their maximum values against the normalized detectors detunings $D_{H}=\left(\omega_{f}-\omega_{0}\right) / \Gamma$ and $D_{M}$, respectively, for fixed values of the parameters $\left(t, \Omega_{0}, k_{0}, j_{0}, k, j\right)$ and the Gaussian pulse parameters $\left(C, \tau_{R}, n\right)$ in the initial vacuum and number states of the $\mathrm{HO}$.

\subsection{Haar Spectrum}

For initial vacuum state $\left(n_{0}=0\right)$, the normalized spectrum $S_{H}\left(D_{H}\right)$ is governed by the quantity $\left|J_{2, H}\right|^{2}$ in the second term in Equation (14). At exact resonance $\left(\Delta_{o}=0\right)$ and for zero shift $k_{o}=0$ and no chirp $(C=0)$, the bi-polar nature of the HW wavelet function reflects itself in the spectrum as two symmetric peaks around $D_{H}=0$ with fading symmetric smaller peaks for one pulse $(n=1)$ case-Figure 1a. Increasing the pulse number $n=2,10$ tends to merge the two peaks of $(n=1)$ case to one central peak that is more narrowed for larger $n \geq 10$ with very small two peaks around this central peak (Figure $1 b$ ). The interplay of the detuning parameter $\left(\Delta_{o} \neq 0\right)$ at large shift parameter $k_{o}=10$ is shown in Figure $1 \mathrm{~b}$, where for one pulse $(n=1)$, the spectrum is asymmetric (one large peak near $D_{H}=0$ and very small flat peak near $D_{H} \sim \Delta_{o}+k_{o}$ ). With increasing pulse number $(n=10)$, the spectrum has a central narrowed peak shifted to the right, $D_{H}>0$ with dense oscillations in its envelope and two flattening asymmetric peaks. For non-zero chirp parameter $(C \neq 0), \Delta_{o}, k_{0} \neq 0$, the asymmetry is more pronounced for $c \gtrless 0$, with very dense oscillatory envelopes for the main peak shifted for $D_{H}>0$ with $C>0$ (Figure 2a) or the main peak around $D_{H} \sim 0$ with $C<0$ (Figure $2 b$ ).

For initial number state $\left(n_{0}=1\right)$, the combination of the three parameters $\left(\Delta_{0}, k_{0}, C\right)$ is best seen with larger pulse number $(n=10)$ and large $k_{0}$. In Figure 3, curve (a), for $\left(\Delta_{o}=3, k_{o}=10, C=0\right)$ there is a single narrowed asymmetric peak to the right of $D_{H}=0$ with two asymmetric broader peaks. The non-zero chirp $C>0$, causes the spectrumFigure 3, curve (b), to have two main peaks asymmetrically displaced from $D_{H}=0$, with small spikes in between. The case of $C<0$-Figure 3-curve (c), makes the spectrum less asymmetric, with central spike near $D_{H}=0$. 
Therefore, the oscillations in the Haar WT spectrum with initial vacuum state are due to non-zero values of atomic detuning $\left(\Delta_{o}\right)$ and the window shift parameter $k_{o}$. These oscillations become denser with large pulse number $(n \geq 10)$ and non-zero chirp parameter $(c \neq 0)$ Figure $2 \mathrm{a}, \mathrm{b}$ and they are diffused in the case of initial number state $\left|n_{0}\right\rangle$ (the term promotional to $n_{0}$ in Equation (14), Figure 3).


Figure 1. (a) The normalized Haar spectrum $S_{H, o}\left(D_{H}\right)$ against the normalized detuning $D_{H}$ with initial vacuum state for $j_{o}=1, k_{o}=0, \tau=\tau_{1}-k_{o} 2^{-j_{o}}, \tau_{1}=2 \pi, \tau_{R}=1, \tau_{o}^{\prime}=1, C=0, \Delta_{o}=0$ and for pulse number $n=1,2,10$ (b) As (a) but for $\Delta_{o}=3, k_{o}=10$. 

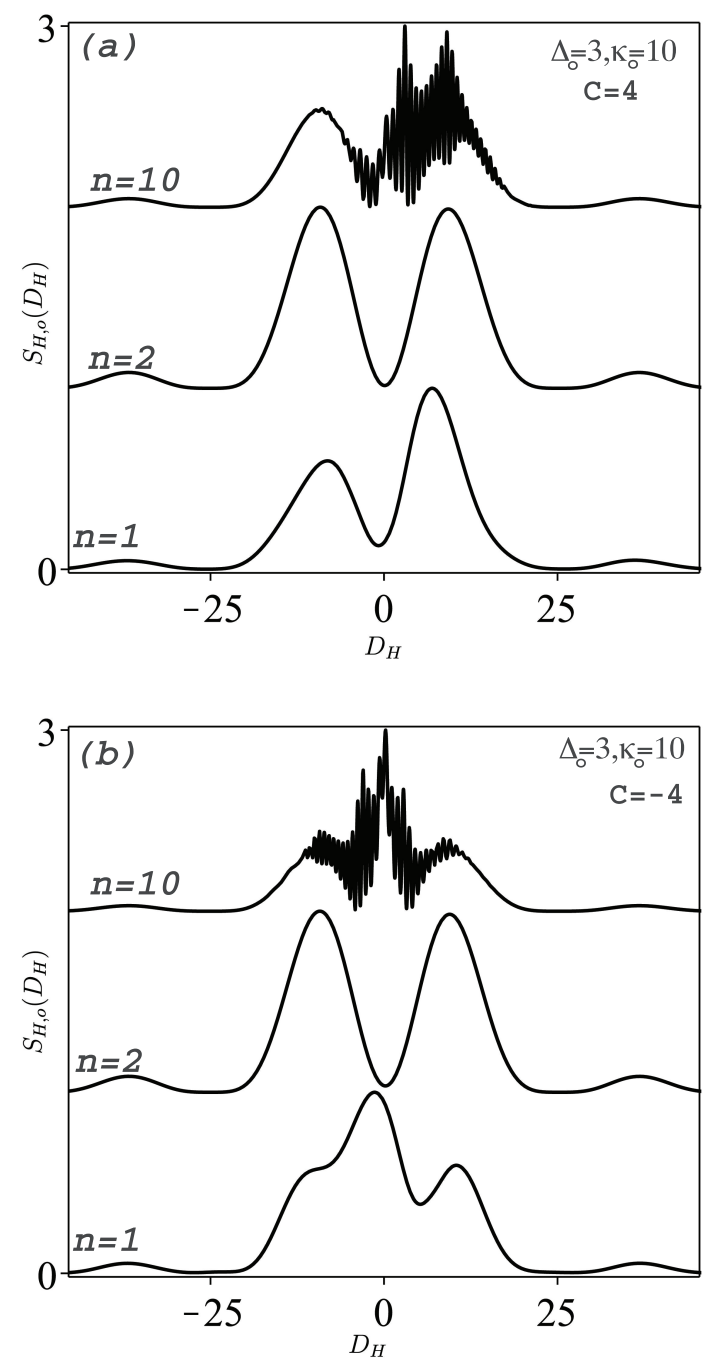

Figure 2. As Figure 1 but for $\Delta_{o}=3, k_{o}=10$ and for: (a) As (b) but for $C=4$ (b) As (b) but for $C=-4$.

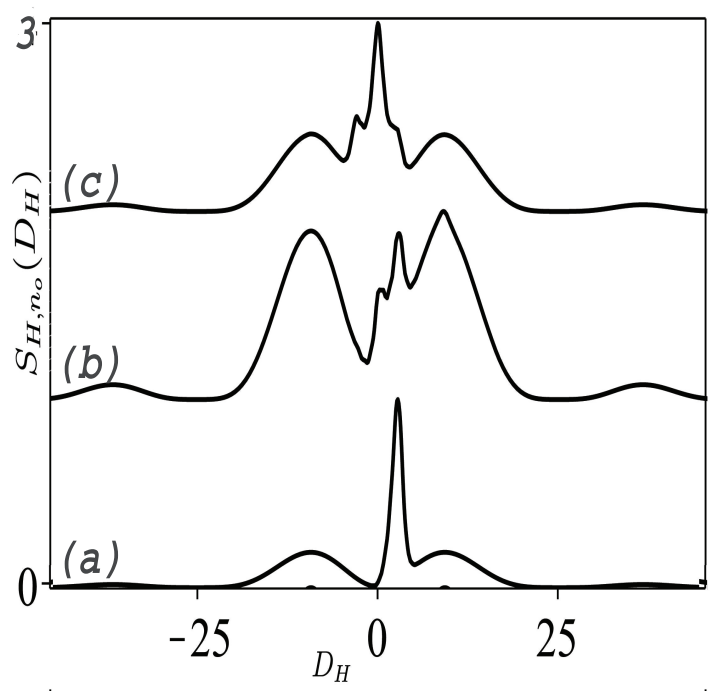

Figure 3. (a) The normalized spectrum $S_{H, n_{0}}\left(D_{H}\right)$ against the normalized detuning $D_{H}$ with initial number state $\left(n_{o}=1\right)$ for $j_{o}=1, k_{o}=10, \tau=\tau_{1}-k_{o} 2^{-j_{o}}, \tau_{1}=2 \pi, \tau_{R}=1, \tau_{o}^{\prime}=1, C=0, \Delta_{o}=3$, $\Omega_{o}=0.01$ and for pulse number $n=10$. (b) As (b) but for $C=4$ (c) As (b) but for $C=-4$. 


\subsection{Morlet Spectrum}

For initial vacuum state $\left(n_{0}=0\right)$, at exact resonance, the general feature of the Morlet (Gaussian) wavelet spectrum is a single symmetric peak, Figure $4 a$, that turns to dense oscillatory spikes due to the large shift parameter $k=10$-Figure $4 \mathrm{~b}$. For large $n=5$ pulse, and non-zero chirp $C=6$, there is a main peak at $D_{M}=0$ with irregular and asymmetrical oscillatory envelopes, Figure 4c.

For initial number state $\left(n_{0}=5\right)$, and weaker Rabi frequency $\left(\Omega_{0}=1\right)$ and large pulse $n=5$, in the off-resonance case $\left(\Delta_{o}=10\right)$ the spectrum is a skewed single peak with little grooving on its envelope for $D_{M}>0$, Figure 5 .

Therefore, the Morlet WT spectrum and with initial vacuum state and single pulse $(n=1)$ has dense oscillatory structure due to large shift parameter of the Morlet window function, Figure $4 \mathrm{~b}$ or irregular oscillations due to large pulse number and non-zero chirped parameter.
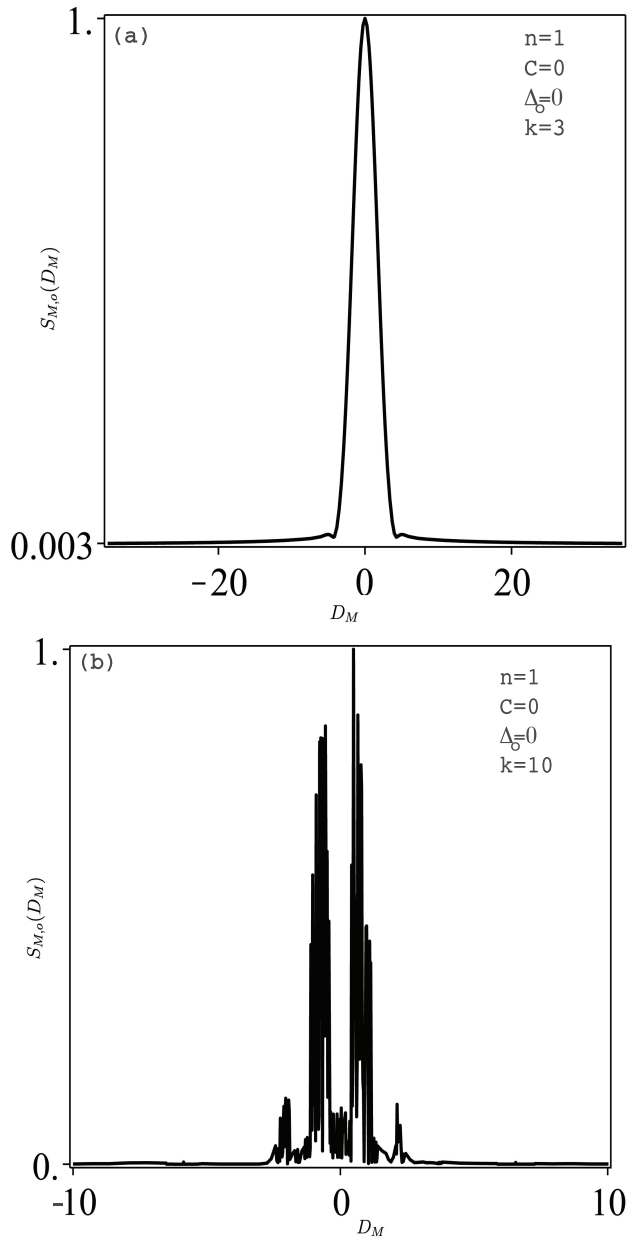

Figure 4. Cont. 


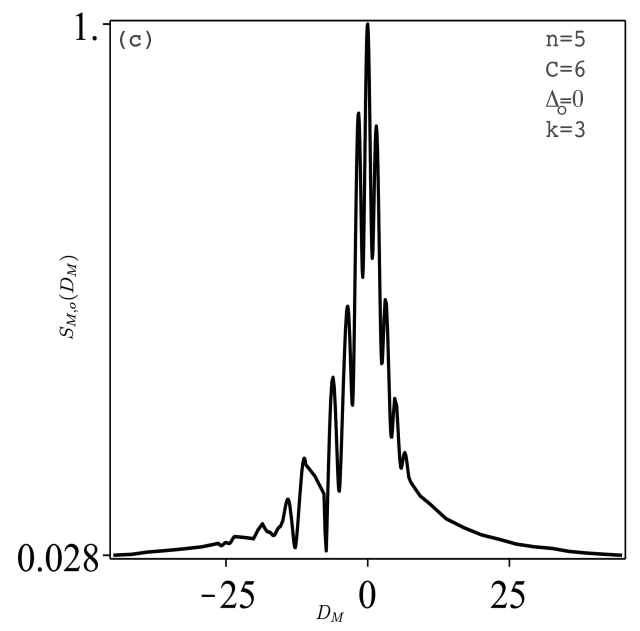

Figure 4. The normalized Morlet spectrum $S_{M, o}\left(D_{M}\right)$ against the normalized detuning $D_{M}$ with initial vacuum state, for $j=1, t=1, \Gamma=0.1$ and for: (a) $n=1, C=\Delta_{o}=0, k=3$. (b) As (a) but with $k=10$. (c) $n=5, C=6, \Delta_{o}=0, k=3$.

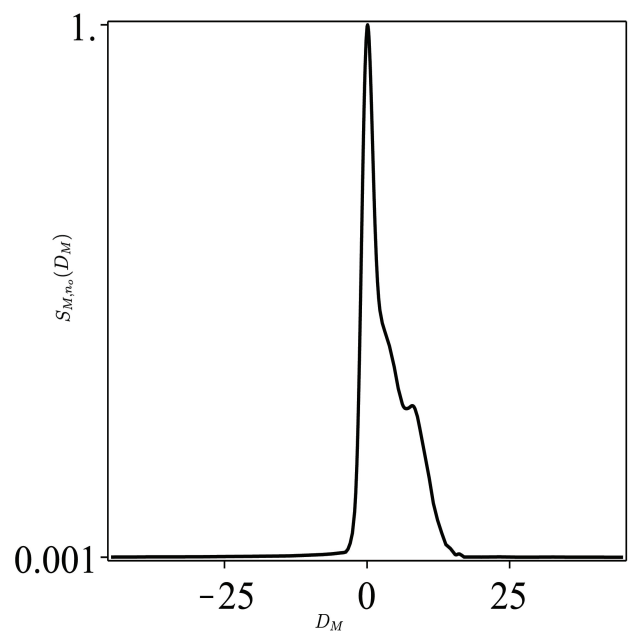

Figure 5. The normalized spectrum $S_{M, n_{o}}\left(D_{M}\right)$ against the normalized detuning $D_{M}$ with initial number state $n_{o}=5$ for $k=3, j=1, t=1, \Gamma=0.1, C=2, \Omega_{o}=1, n=5, \tau_{R}=0.5, \Delta_{o}=10$.

\section{Summary}

The time-dependent wavelet spectra of the fluorescent radiation due to the coupled system of non-dissipative harmonic oscillator $(\mathrm{HO})$ with a train of chirped Gaussian pulses are calculated analytically and investigated computationally using two wavelet window functions: the Haar and Morlet wavelet functions. Asymmetry, narrowing and dense oscillations in the spectra in the two cases of initial and number states of the $\mathrm{HO}$ are essentially associated with large shift parameter, non-zero chirp and large pulse numbers. In general, these features are absent in the Fourier transform spectrum [5], due to the averaging technique over the entire time interval of the detected signal (c.f. [9]). Study of pulsed-driven qubit system is very essential within the context of quantum information sciences and quantum computing. In particular, the spectrum function of the scattered radiation provides information about the splitting of the atomic energy levels, so the response of the atom for a certain frequency can be identified [20]. Finally, it is worth mentioning physically that the driven $\mathrm{HO}$ realized as radiation-radiation interaction may be also realized as radiation-matter interaction, with matter sought as $N \rightarrow \infty$ number of atoms bathed in a thermal or squeezed vacuum radiation [21-23]. Therefore, our results provide deep insight into the giant quantum oscillator (formed as noted in this $N \rightarrow \infty$ limit) and driven by the chirped Gaussian laser pulses. 
Author Contributions: R.A.A. and H.A.; formal analysis, H.A.; visualization. R.A.A.; writingoriginal draft preparation. All authors have read and agreed to the published version of the manuscript.

Funding: This research received no external funding.

Institutional Review Board Statement: Not applicable.

Informed Consent Statement: Not applicable.

Data Availability Statement: Not applicable.

Conflicts of Interest: The authors declare no conflict of interest.

\section{References}

1. Mandel, L.; Wolf, E. Optical Coherence and Quantum Optics; Cambridge University Press: Cambridge, UK, 1995.

2. Walls, D.F.; Milburn, G.J. Quantum Optics; Springer: Berlin, Germany, 2007.

3. Joshi, A.; Hassan, S.S. Resonance fluorescence spectra of a two-level atom and of a harmonic oscillator with multimode rectangular laser pulses. J. Phys. B At. Mol. Opt. Phys. 2002, 35, 1985-2003. [CrossRef]

4. Hassan, S.S.; Alharbey, R.A.; Al-Zaki, H. Transient spectrum of $\sin ^{2}$-pulsed driven harmonic oscillator. J. Nonlinear Opt. Phys. Mater. 2014, 23, 1450052. [CrossRef]

5. Hassan, S.S.; Alharbey, R.A.; Jarad, T.; Almaatooq, S. Driven harmonic oscillator by train of chirped Gaussian pulses. Int. J. Appl. Math. 2020, 33, 59-73. [CrossRef]

6. Hassan, S.S.; Alharbey, R.A.; Jarad, T. Transient Spectrum of Pulsed-driven Harmonic Oscillator: Damping and Pulse Shape Effects. Nonlinear Opt. Quantum Opt. Concepts Mod. Opt. 2018, 48, 277-288.

7. Hassan, S.S.; Alharbey, R.A. Fourier and Haar Wavelet Spectra of a Driven Harmonic Oscillator without Rotating Wave Approximation. Nonlinear Opt. Quantum Opt. Concepts Mod. Opt. 2019, 50, 315-325.

8. Hassan, S.S.; Alharbey, R.A.; Matar, G. Haar wavelet Spectrum of $\sin ^{2}$-pulsed driven harmonic oscillator. Nonlinear Opt. Quantum Opt. 2016, 48, 29-39.

9. Addison, P. The Illustrated Wavelet Transform Handbook; CRC Press: Boca Raton, FL, USA, 2017.

10. Mohamed, A.A.; Hassan, S.S.; Al Saegh, M.A. Wavelet spectra of scattered radiation with pulsed driven system. Nonlinear Opt. Quantum Opt. Concepts Mod. Opt. 2007, 36, 107-116.

11. Hassan, S.S.; Al-Saegh, M.A.; Mohamed, A.S.; Batarfi, H.A. HaarWavelet Spectrum of a Pulsed-Driven Qubit. Nonlinear Opt. Quantum Opt. Concepts Mod. Opt. 2011, 42, 37.

12. Batarfi, H.A.; Hassan, S.S. Haar wavelet spectrum of an exponentially pulsed driven qubit. Nonlinear Opt. Quantum Opt. Concepts Mod. Opt. 2017, 48, 93-105.

13. Alharbey, R.A. Haar wavelet spectrum of sin2-pulsed driven qubit. Optik 2016, 27, 9878-9884. [CrossRef]

14. Louisell, W.H. Quantum Statistical Properties of Radiation; John Wiley: New York, NY, USA, 1973.

15. Heffner, H.; Louisell, W.H. Transformation Having Applications in Quantum Mechanics. J. Math. Phys. 1965, 6, 474-478. [CrossRef]

16. Lebedev, N.N. Special Functions and Their Applications; Prentice-Hall: Dover, NY, USA, 1972.

17. Eberly, J.H.; Wodkiewicz, K. The time-dependent physical spectrum of light. JOSA 1977, 67, 1252-1261. [CrossRef]

18. ANajmi, A.H. Wavelets: A Concise Guide; John Hopkins Univ. Press: Baltimore, MD, USA, 2012.

19. Boggess, A.; Narcowich, F. A First Course in Wavelets with Fourier Analysis; Prentice-Hall: Hoboken, NJ, USA, 2001.

20. Hassan, S.S.; Joshi, A.; Al-Madhari, N.M. Spectrum of a pulsed driven qubit. J. Phys. B At. Mol. Opt. Phys. 2008, 41, $145503-145507$. [CrossRef]

21. Hassan, S.S.; Hildred, G.P.; Puri, R.R.; Bullough, R.K. Incoherently driven Dicke model. J. Phys. B At. Mol. Opt. Phys. 1982, 15, 2635-2655. [CrossRef]

22. Bullough, R.K.; Puri, R.R.; Hassan, S.S. Some Remarks on the Organisation of Living Matter and Its Thermal Disorganisation. In Molecular and Biological Physics of Living Systems; Springer: Dordrecht, The Netherlands, 1990; pp. 1-18.

23. Wahiddin, M.R.; Hassan, S.S.; Bullough, R.K. Cooperative atomic behaviour and oscillator formation in a squeezed vacuum. J. Mod. Opt. 1995, 42, 171-189. [CrossRef] 